\title{
Temperature and Refractive Index Sensing Based on Plasmonic Fano Resonance
}

\author{
Sharmistha Chatterjee ${ }^{1,2}$, Giovanna Palermo ${ }^{2}$, Theodore Letsou ${ }^{1}$, Giuseppe Lio ${ }^{2}$, Antonio De Luca ${ }^{2}$, \\ Giuseppe Strangi ${ }^{1,2}$ \\ ${ }^{1}$ Department of Physics, Case Western Reserve University \\ 10600 Euclid Avenue, Cleveland, Ohio 44106, USA. \\ Sxc1211@case.edu \\ ${ }^{2}$ CNR-NANOTEC Istituto di Nanotecnologia and Department of Physics, University of Calabria \\ 87036-Rende, Cosenza, Italy
}

\begin{abstract}
We report experimental demonstration of a Fano type resonant structure for simultaneous realization of change in external temperature and refractive index. The Fano resonant structure has been made by depositing a hybrid nano-composite on silver thin film. The study has also been done by adding a dielectric silica spacer layer between the hybrid nano-composite and silver thin film. In the hybrid nano-composite plasmonic gold nanorods are synergistically integrated with Pnipam, a thermo-responsive polymer. Here Fano resonance arises from the near field coupling of narrow localised surface plasmon (LSP) mode of gold nanorods and broad surface plasmon (SP) mode of silver thin film. The coupling strength and thus the Fano line shape which depends on the separation between the two plasmonic systems is seen to be changed by incorporating the dielectric silica spacer layer and by changing the hydrodynamic diameter of the Pnipam with the variation of the external temperature. This indicates that the mentioned hybrid system has the capability to sense change in external temperature and refractive index simultaneously. Furthermore we have done numerical investigation based on finite element method (FEM) to see the effect on coupling of gold nanorods and silver thin film upon changing the separation distance between them which is giving an idea about the Fano strength variation. All these are suggesting that this system will not only help for the label-free detection of the foreign protein molecule but also for identifying the different thermodynamic states and reactions of the molecule which is crucial for protein engineering.
\end{abstract}

Keywords: Fano resonance, LSP, SP, FEM.

\section{Introduction}

Localized surface plasmon resonances (LSPRs) arising from the collective electron oscillations in metal nanoparticles (MNPs) have attracted tremendous interests for its ability to concentrate and manipulate light in nanoscale regime. The strong electromagnetic field enhancement arising due to the LSPR can boost many linear and non-linear optical processes and is useful in different practical applications [1]. But recently Fano resonance in plasmonic systems have attracted a lot of attention compared to the symmetric Lorentzian characteristic lineshape supported by the LSPR of MNPs due to its useful properties such as a narrow asymmetric line-shape, strong electromagnetic field enhancement, light confinement to the nanometer scale, and high spectral sensitivity to changes in the local dielectric environment [2]. The Fano resonances, identified by their characteristic asymmetric lineshape were first discovered in atomic systems, experiencing interference between discrete and continuum states. Analogously Fano resonances in plasmonic systems originate from the interference between a bright mode (superradiant), and a dark mode (subradiant) via near field coupling. A Fano peak is a consequence of constructive interference between such modes, whereas a Fano dip arises as the two modes interfere destructively. Taking advantage of this principle, different types of plasmonic structures, such as metamaterials, clusters, single and multi-surface nanoparticles and periodic structures have been proposed and studied, seeking Fano resonances in their spectral responses [2]. Structures generating pronounced Fano resonances include assemblies of plasmonic nanoparticles, such as dimmers, trimers, quadrumers, pentamers and heptamers, where various parameters such as inter-particle separation and particle size can be tuned, but also symmetry can be broken, to tailor the characteristics of Fano resonances [2]. Because of the pronounced asymmetric spectral lineshape and highly enhanced local fields, plasmonic Fano resonance can be effectively exploited for applications ranging from chemical and biological sensors, to surface enhanced Raman 
spectroscopy (SERS) and nonlinear optics. Realizing the advantages of Fano line shape over the Lorentzian shaped resonance many research groups have used that for label-free detection of protein molecule with high sensitivity [3]. A group of researchers have also used Fano line shape for external temperature sensing [4]. But there is no report in the literature about a Fano resonant structure for measuring the change in both external temperature and refractive index simultaneously. Here we have experimentally demonstrated a system whose characteristic lineshape is of Fano type and it can efficiently respond to the change in both external temperature and refractive index simultaneously. Relevant numerical investigation has also been done based on finite element method here. All these are indicating that this kind of system will not only help for the label-free detection of the protein molecule but also for identifying the different thermodynamic states and reactions of the molecule which is crucial for protein engineering.

\section{Results and Discussion}

We have experimentally observed Fano resonance in our plasmonic system which is not only useful for refractive index sensing but also external temperature sensing simultaneously. A schematic diagram of the system we have used is shown in figure 1.

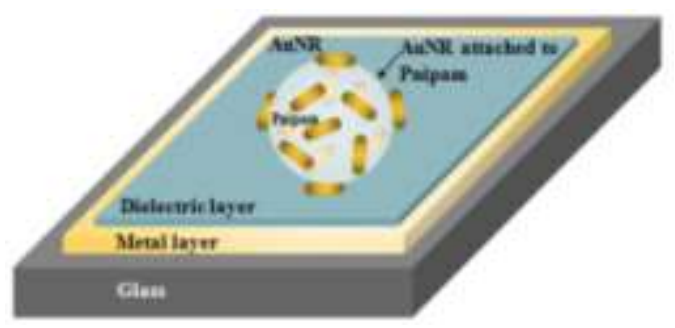

Fig. 1: Schematic diagram of the plasmonic system we have used.

Here, Au nanorods (AuNRs) are attached on the surface of a Pnipam polymer which is separated from $80 \mathrm{~nm} \mathrm{Ag} \mathrm{thin}$ film by $\mathrm{SiO}_{2}$ dielectric spacer layer. Fano resonance is originating here from the hybridization of the localized surface plasmons (LSPs) of AuNRs and surface plasmons (SPs) of Ag thin film. This fano line shape can be tuned by varying the thickness of the spacer dielectric layer which has been shown in figure 2.

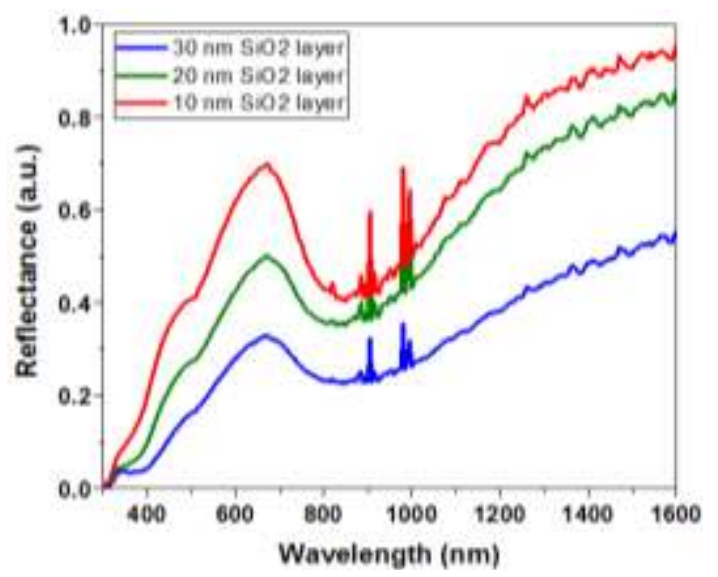

Fig. 2: Variation of Fano line shape for different dielectric spacer layer thickness in room temperature.

The external temperature variation also can tune this fano lineshape. Figure 3 is showing how external temperature variation affects the Fano line shape. All these reflectance measurement have been done using a V-vase ellipsometer and the used Heater is a Calctec Hs unit which controls the external temperature. Pnipam which is a temperature sensitive 
polymer is holding the AuNRs on its surface. In room temperature, the hydrodynamic diameter of the Pnipam ball is approximately $544 \mathrm{~nm}$ while that becomes $306 \mathrm{~nm}$ upon increasing the temperature beyond $40^{\circ} \mathrm{C}$. If the external temperature changes then the hydrodynamic diameter of the Pnipam gets changed and it affects the inter-distance (reversible change) and thus interaction between different AuNRs and between AuNRs and the Ag film. Because of this reason the fano resonance arising here from the near field coupling of AuNRs and Ag film can be tuned by change in external temperature. Here Pnipam Samples has been prepared via a well known route [5] and to prepare our plasmonic system we have drop-casted $12 \mu \mathrm{l}$ of Pnipam sample on $80 \mathrm{~nm} \mathrm{Ag}$ metal film (with and without $\mathrm{SiO}_{2}$ layer). As this Pnipam sample has two stable thermodynamic states it can only sense these two temperature state. Instead of taking Pnipam if one takes any other polymer which can change their property over broad range of temperature (or any other physical parameters like stress, strain etc) continuously then that system may give response for a slight change in the external temperature. But this kind of Pnipam is good for protein state identification as protein has similar kind of coil to globule transition property.

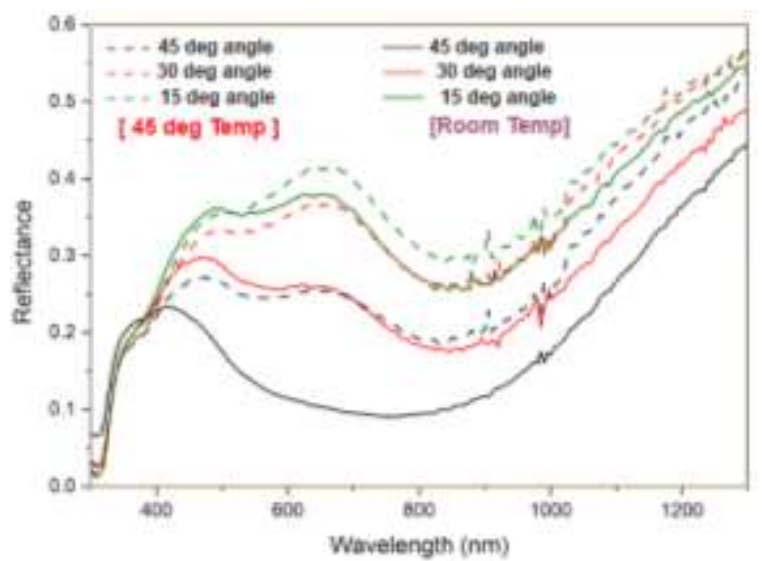

Fig. 3: Variation of Fano line shape for varying temperature

Furthermore, we have done numerical investigation based on FEM to see the effect on coupling of AuNRs and Ag thin film upon changing the dielectric spacer layer thickness which is giving an idea about the Fano strength. The numerical investigation is described in figure 4 which can be correlated with the experimental results about the Fano strength for different spacer layer thickness described in figure 2. From the figure 4 it is very clear that the plasmonic field strength in the spacer layer is higher for $10 \mathrm{~nm} \mathrm{SiO}$ layer than the $30 \mathrm{~nm} \mathrm{SiO}$ layer which can be verified from the experimental results. As the system is 3D symmetric, during FEM simulations it has been analyzed in 2D axisymmetrically to minimize the calculation time. The wavelength dependent optical constants of metals are taken from the Johnson and Christy measurements.

So, all these results are indicating the usefulness of this fano lineshape for sensing the refractive index of the surrounding media as well as the temperature which makes this system as a unique one. Due to the extreme intensity enhancement in the spacer layer (because of the gap plasmons) and the unique line shape of fano, detection of protein molecule (in the spacer layer) in ultralow concentration with high $\mathrm{S} / \mathrm{N}$ ratio could be possible comparatively easily with this system with an additive advantage of having temperature sensitiveness. 

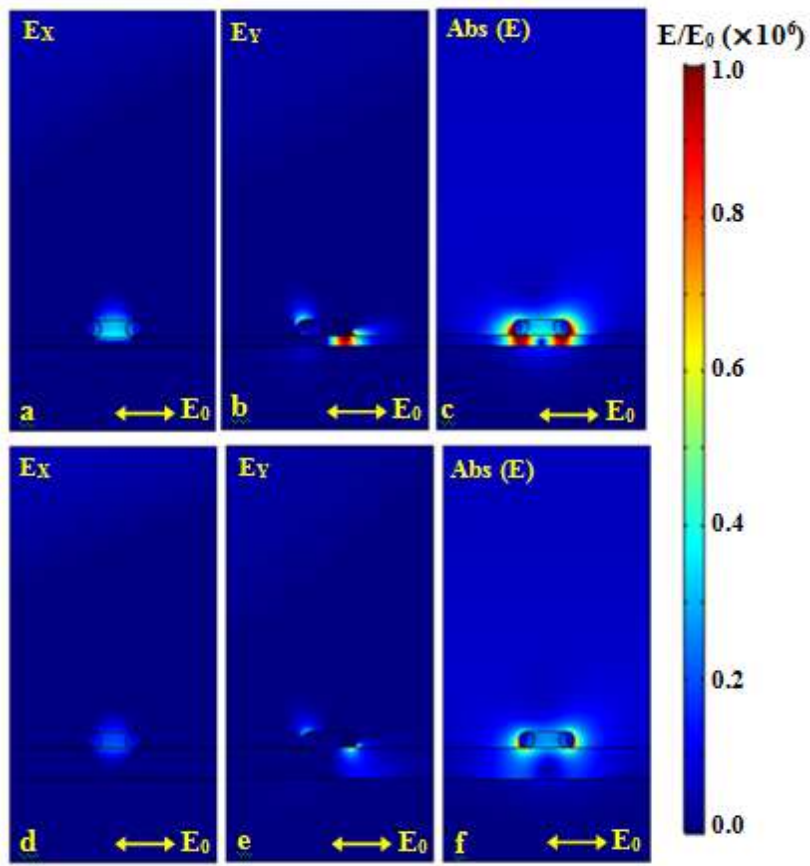

Fig.4: FEM simulations for electric field mapping. Panel a, b, c are describing the electric field enhancements for case of 10 nm $\mathrm{SiO}_{2}$ spacer layer whereas panel d, e, f are describing that for case of $30 \mathrm{~nm} \mathrm{SiO}{ }_{2}$ spacer layer. Every calculation is done for 30 deg incidence angle. The asymmetry in the electric field is pointing out towards its Fano nature. One can clearly see a decrease in the electric field strength in the spacer layer for $30 \mathrm{~nm} \mathrm{SiO} 2$ with respect to $10 \mathrm{~nm} \mathrm{SiO}$ layer. This is occurring because of the decrease in the coupling strength for $30 \mathrm{~nm} \mathrm{SiO}_{2}$ layer.

\section{Conclusion}

In summary, we have experimentally demonstrated a system whose characteristic line shape is of Fano type and is useful for not only to detect change in external refractive index but also temperature change, making this response useful for many application and specially for protein engineering.

\section{References}

[1] J. A. Schuller, E. S. Barnard, W. S. Cai, Y. C. Jun, J. S. White, and M. L. Brongersma, "Plasmonics for Extreme Light Concentration and Manipulation," Nat. Mater., vol. 9, pp. 193-204, 2010.

[2] B. Luk'yanchuk, N. I. Zheludev, S. A. Maier, N. J. Halas, P. Nordlander, H. Giessen, and C. T. Chong, "The Fano Resonance in Plasmonic Nanostructures and Metamaterials," Nat. Mater., vol. 9, pp. 707-715, 2010.

[3] B. Gallinet, and O. J. F. Martin, "Refractive Index Sensing with Subradiant Modes: A Framework To Reduce Losses in Plasmonic Nanostructures," ACS Nano, vol. 7, no. 8, pp. 6978-6987, 2013.

[4] Y. Kong, Q. Wei, C. Liu, and S. Wang "Nanoscale temperature sensor based on Fano resonance in metal-insulatormetal waveguide," Opt. Comm., vol. 384, pp. 85-88, 2017.

[5] Y. N. Montecelo, M. C. Hermo, X. T. Kong, B. R. González, Z. Wang, M. P. Lorenzo, A. O. Govorov, and M. A. C. Duarte, "Traveling hot-spots in plasmonic photocatalysis: Manipulating interparticle spacing for real-time control of electron injection," ChemCatChem., vol. 10, pp. 1-7, 2018. 